\title{
Domestic Studies as a Foundation for Progression of National Mental Health
}

\author{
Saeed Shoja Shafti* \\ Professor of Psychiatry, University of Social Welfare and Rehabilitation Sciences (USWR), Iran
}

*Corresponding author: Saeed Shoja Shafti, Professor of Psychiatry, University of Social Welfare and Rehabilitation Sciences (USWR), Razi Psychiatric Hospital, Tehran, Iran.
Received Date: March 31, 2019

Published Date: April 17, 2019

\section{Short Communication}

Systematic studies are precise tools for finding practical solutions with respect to different aspects of human's life troubles. So, regarding this issue, mental health as well cannot be treated as a discrete unit, especially since it includes the full spectrum of human behavior. Although supposition of countless diversities among low, middle and high-income societies are reasonable, but there is also some shortfalls that are not due to lack of revenues, but due to unsuitable plans taken by researchers in such countries. For example, absence of low-income countries in the field of mental health's research is obviously notable. Accepting indisputably the findings of high-income societies in the field of mental health cannot always contain alike favorable resonance throughout different societies, especially regarding cultural aspects. CulturalHistorical stage of development of every society will filter automatically exposed data, based on its societal - habitual thresholds and practical significance of facts. Modern epoch demands mobilization of assets in the way of promoting autonomy of low and middle-income societies in resolving their mental health problems based on their own scientific efforts, instead of concrete repetition of perspectives of dissimilar societies. For example, while in the field of psyopharmacotherapy the issue of pharmacogenetic, with its pertaining pharmacokinetic and pharmacodynamic implications, necessitates genuine nationalized studies with respect to their specific native target groups, procedures like psychotherapy, which can include a lot of cultural variables, surely may perhaps necessitate additional insight and amendment by native practitioners. Psychoanalysis, psychoanalytic psychotherapy, cognitive therapy, behavior therapy, cognitive behavior therapy and so on, are not utilizable evenly in different civilizations, not merely due to lack of skills and training, but many times owing to nonacceptance or lack of sufficient tolerance, based on community values. Such a contradiction between science and culture in the field of psychiatry and psychotherapy cannot be resolved without active intervention of local researchers for lessening the distance between presented facts and suitable practices, by taking into account the hidden hindrances and inner resistances of clientele. Careful understanding of people's attitude regarding the suggested or employed methods is not a hard task, but requires firstly enough concern, and secondly sufficient awareness of the current society's intervening parameters. Unconditional acceptance of DSM and ICD classifications, which are certainly established on a comprehensive collection of longitudinal and cross-sectional information, cannot relinquish responsibility with respect to validation of its diverse components by other societies that want to employ it. Specificity and sensitivity of diagnostic criteria in the realm of descriptive and phenomenological approach, which is based generally on algebraic analysis of symptoms, certainly can gather more confidence from more universal studies. Plenty of unspecified diagnoses, culturebound syndromes, employment of different diagnostic categories by different examiners, different outlooks between DSM and ICD, and in particular considering all of these debates disrespect to etiology, stress once more on this issue that replying to these controversies cannot neglect the native psychosocial variables. The end result of such ignorance cannot be anything except than copying the modern society's studies disregard to their necessity or priority in the context of their own society's troubles. It is interesting that in many low or middle income nations there is not hitherto any accurate statistical assessment with respect to the prevalence and incidence of mental disorders and their estimation depends on high- income society's proportions; But nevertheless in the same countries there is a lot of interest to theoretical aspects of behavioral sciences, disregard to their chronological process of development. Such a blind course of action can displace easily the foremost priorities with less important ones and mobilize assets ineffectually. Meanwhile different political, social and economical troubles usually precede the importance of mental health in countries where 
priority is defined according to the resolution of immediate and sensible vital needs. In such circumstances mental health can be pushed back as far as possible in the hierarchy of 'primary health cares' by ignoring the importance of psychopathology in creation of a large cluster of social troubles. Additionally, extreme accentuation on the importance of biological psychiatry and scientific neuroscience has caused the illusion that firstly psycho-social studies are less important than biological ones and secondly since biological studies are dependent on modern and profound technology therefore lack of such instruments already prohibit accomplishment of such trials in developing societies. Surely such a judgment needs revision. Every society, even the poorest one, can have or provide a minimum of equipment's and workforces for accomplishment of some purposeful studies. Confined and small but specialized and meticulous investigations are attainable provided that based on well-defined short-term and long-term goals. Besides and based on day-to-day problems of daily life, clinical and practical studies, in essence, have priority on researches in the field of basic sciences in developing or non-developed countries. Integration of different sections and services, instead of specification, also had been encouraged for economizing of budget and staff in low and middle- income societies. All of these strategies, at a national level, can promote public studies more feasible than before. At this time, mental health is so extended that a clinician cannot do at the same time both of practice and research as complete as possible. It seems that independent research sections are necessary for employment of trained and skillful researchers to follow sequential and great beneficial investigations instead of scattered and non-systematic researches that usually take far from replying to major social dilemmas. On the other hand, due to traditional and spiritual believes usually there is a large interest in alternative medicine in different societies. Such interests are similar in different cultures except that their prevalence among common people differs according to the intellectual and cultural background. Magic-seeking tendencies of human creature reinforce some researches to be involved in such old-fashioned methods, which's frequent shortfall through history has pushed authentic science ahead. Such problem, which seems to be more prevalent among people of low and middle-income societies, can waste a large energy that deserves to be running into the valid and reasonable channels. For that reason, scientific evolution generally cannot be considered free from cultural development. Unfortunately, even now in many societies behavioral sciences are regarded as a subdivision of humanistic sciences, something like law, literature and sociology. This kind of approach keeps mental health out of its non-spiritualistic, non-philosophical aspects. Inclusion of psychosocial factors in human behavior is decoding by many as spiritualistic nature of human being, and not the continuing environmental influence. This misunderstanding had a detrimental effect on imagination of scientific mental health, because cannot separate an ingrained internal need from an external fact, a trouble that can confound the process of research as well. So, it seems that research-related strategies in low and middle-income countries, in the field of mental health, needs a basic revision because persistence of such passive attitudes cannot guarantee finding of better answers for their specific problems. Determining well-defined goals can only be attained after delineating primary national data. Such a process is necessary for portraying an acceptable psychopathological profile in the context of every society. Although pursuing worldwide interests are not deniable, but it cannot disregard the priority of local dilemmas and researches.

\section{Acknowledgement}

None.

\section{Conflict of Interest}

No conflict of interest. 